\title{
Oficina de formação sobre moocs para promover novas oportunidades
}

\section{Vitor Gonçalves ${ }^{a}$}

${ }^{a}$ Centro de Investigação em Educação Básica, Instituto Politécnico de Bragança, Bragança, Portugal, vg@ipb.pt.

\section{Resumo}

Os Cursos Online Abertos e Massivos (Massive Open Online Courses) correspondem, hoje em dia, a uma das mais recentes oportunidades para os professores investirem na sua formação contínua, normalmente em modalidade informal, com o objetivo de melhorar ou atualizar conhecimentos e habilidades nas suas áreas cientificas, mas também como um complemento ao seu posto de trabalho enquanto professores ou formadores. Nesta perspetiva, eles próprios podem ser os profissionais de educação que contribuem para melhorar a qualidade e a disponibilidade de cursos deste tipo em língua portuguesa. Para tal, foi revisitado um plano de um curso de formação a distância, já anteriormente organizado na modalidade de oficina de formação online, desenvolvida uma versão atualizada do mesmo, bem como do correspondente "MOOC sobre MOOCs e outras tecnologias educativas" que suportaria parte desta nova formação contínua de professores. Inscreveram-se 69 formandos, tendo sido constituídas três turmas de 20 elementos cada para frequentarem de forma síncrona as 25 horas da oficina de formação. Avaliada a sua participação, podemos concluir dois aspetos principais: a participação numa oficina de formação deste tipo exige automotivação e imbricação por parte dos formandos, o que explica a desistência de 26 formandos ao longo da oficina de formação. Dos 34 formandos que concluiram esta oficina, os aspetos que se destacaram foram as dificuldades em conciliar a formação com o trabalho laboral, apesar de todos sem exceção enfatizarem as potencialidades desta modalidade de formação e dos resultados que a mesma poderá vir a proporcionar num futuro próximo.

Palavras-chaves: MOOC, oficina de formação, formação contínua, professores. 


\section{Introdução}

Os Cursos Abertos Online Massivos ou, em inglês, Massive Open Online Courses, comummente conhecidos por MOOC, podem constituir uma tecnologia educativa para fomentar a formação a distância, possibilitando a qualquer indivíduo adquirir um conjunto de conhecimentos que lhe podem vir a ser úteis no exercício da sua prática profissional. Os MOOC podem assim assumir-se como uma poderosa e emergente estratégia de aprendizagem, quer como complemento aos processos de educação (in)formais, quer na área da formação contínua de professores. Focando-nos nesta área de formação profissional, os MOOC podem constituir uma oportunidade para os professores investirem na sua formação contínua, independentemente das características espácio-temporais e de todas as condicionantes próprias da carreira docente. Acredita-se que os professores se podem desenvolver profissionalmente através dos MOOC, melhorando ou atualizando os seus conhecimentos e as suas competências, tal como através da formação presencial. Apesar dos MOOC poderem mediar ou até mesmo suportar a formação contínua de professores, não podemos deixar de referir que a formação presencial continua a ser essencial para diversas tipologias de formação e de formandos e para situações específicas cujas características exijam processos de ensino e aprendizagem presenciais.

Em 2015, foi planeado, desenvolvido, distribuído e utilizado um MOOC, denominado "MOOC sobre MOOCs e outras tecnologias educativas", através da plataforma Udemy e destinado a suportar uma oficina de formação contínua de professores denominada MOOC: uma tecnologia educativa do futuro. Participaram nesta edição do curso dezoito profissionais, tais como, educadores de infância e professores dos ensinos básico e secundário, de áreas e graus académicos distintos. Destes, concluíram com aproveitamento 15 formandos.

No início de 2018, este MOOC foi revisitado no sentido de o atualizar e melhorar de acordo com os resultados e recomendações da avaliação inerente à primeira edição e, assim, contribuir para melhorar a qualidade e a disponibilidade de cursos deste tipo em língua portuguesa. Consequentemente, a segunda edição da oficina de formação "MOOC: uma tecnologia educativa do futuro" teve início a março de 2018. Assim, no presente trabalho, adotou-se a metodologia de estudo de caso com vista a aferir o processo de planeamento, desenvolvimento, utilização e avaliação no âmbito da $2^{\text {a }}$ edição deste MOOC pelos 34 formandos que concluíram a formação. 


\section{Revisão da literatura}

A revisão da literatura procura contextualizar as duas principais temáticas envolvidas neste estudo, nomeadamente a formação contínua de professores e o MOOC enquanto modelo e tecnologia que pode fornecer o suporte para disponibilizar ambientes com objetos de aprendizagem e serviços que permitam aos professores a (auto)aprendizagem, quer ao nível dos seus próprios processos de formação contínua, quer até mesmo ao nível de disponibilizarem ambientes complementares das formações que lecionam.

\subsection{Formação de professores}

Os docentes devem, não apenas reconhecer que as tecnologias podem ser úteis suportes ao processo de ensino e de aprendizagem, mas também utilizá-las para construir ou disponibilizar novas formas de aprendizagem. Assim, "os professores devem ser parceiros na concepção e dinamização de atividades de TIC e não meros espectadores e executores de tarefas" (Penteado \& Borba, 2000, p. 29).

A massificação da utilização da Internet, em particular das redes digitais de aprendizagem, tem vindo a impulsionar novos espaços digitais e novas formas educação e formação que contribuem para a formação contínua de professores. Torna-se, portanto, fundamental que os docentes acompanhem esta mudança e que, através dela, consigam desenvolver competências, adquirir novos conhecimentos e integrar as TIC na sua prática educativa. Neste sentido, é importante a mobilização dos docentes para a aquisição ou atualização das competências tecnológicas através da realização de ações de formação contínua, que permitam o uso das tecnologias, para que, assim, tenham "a oportunidade de aprender e observar novos métodos de aprendizagem com TIC, compartilhar questões e problemas com outros e explorar novas ideias com especialistas e colegas" (Baylor \& Ritchie, 2002, p. 410). Estas oficinas de formação estão previstas na Portaria n. ${ }^{\circ} 731 / 2009$ de 7 de julho (D. R., 2009) que diz respeito ao Sistema de Formação e de Certificação em Competências TIC para os professores, em Portugal, em exercício de funções nas instituições de educação préescolar e dos ensinos básico e secundário. Este sistema de formação organiza-se em três níveis, nomeadamente: Formação em competências digitais (nível 1); Formação em competências pedagógicas e profissionais com as TIC (nível 2); Formação em competências avançadas em TIC na educação (nível 3).

Cabe, portanto, a cada docente, de acordo com os seus objetivos, as suas competências e a sua cultura profissional, não menosprezando o contexto real em que se insere, selecionar as formações mais adequadas à sua realidade profissional para que, através delas, seja possível melhorar a sua prática com as TIC e, essencialmente, melhorar a qualidade do processo educativo. Os MOOC podem não ter provocado a rutura em nenhum sistema de ensino. No 
entanto, tal como afirmou Rick Levin (exCEO Coursera), embora os MOOCs não tenham provocado disrupção no mercado do ensino superior, eles têm vindo a mostrar-se disruptivos em relação ao mercado de trabalho. O público-alvo principal não é o estudante universitário tradicional, mas os "aprendizes de carreira para a vida toda", ou seja, alguém que pode estar muito além da faculdade e faz cursos online com o objetivo de obter crescimento profissional (Shah, 2018).

\subsection{Massive Open Online Courses}

Em termos genéricos, os Massive Open Online Courses ou MOOC não exigem prérequisitos de acesso e possuem um itinerário aberto e flexível (Codarin, 2012). De acordo com o Edu Trends Report (Monterrey, 2014; Monterrey, 2017), destacam-se outras vantagens como, por exemplo: posicionar a marca e os melhores professores; fortalecer os MOOC como uma ferramenta para captar e atrair estudantes para os programas formais; fornecer uma oferta de programas alternativos e de educação contínua; entrar em novos mercados; e, finalmente, desenvolver um novo modelo económico para abordar os mercados emergentes.

De acordo com Siemens (2013), “os MOOCs representam uma continuidade na tendência em inovação, experimentação e uso da tecnologia iniciada pela aprendizagem à distância e on-line, para oferecer oportunidades de aprendizagem de forma massiva" (Siemens, 2013, p. 5). Littlejohn (2013) afirma que um MOOC pode ser definido como um curso que fornece acesso aberto, baseado num modelo de educação a distância, promovendo uma participação interativa em larga escala (Ma, Lee \& Kuo, 2013) e pode ser um dos modos mais versáteis de oferecer educação de qualidade, especialmente para aqueles que residem em regiões distantes ou desfavorecidas (Daradoumis et al., 2013). Assim, um MOOC é "um curso online (que pode utilizar diferentes plataformas), aberto (gratuito, sem pré-requisitos para participação e que utiliza recursos educacionais abertos) e massivo (oferecido para um grande número de alunos)" (Mattar, 2013, p. 30).

Atualmente, existe convergência na literatura em relação à distinção dos MOOC proposta por Downes (2012), que designa uns por cMOOC e os outros por xMOOC (Watters, 2012). Os cMOOC são centrados nos contextos e correspondem a uma perspetiva conectivista. Baseiam-se numa metodologia colaborativa e "são estruturados a partir da aprendizagem auto-organizada, centrada em obter significado através da experiência em comunidade, utilizando ferramentas de participação como blogs, feeds RSS e outros métodos descentralizados" (Torres, 2013, p. 66). Destacam-se alguns exemplos de cursos desta tipologia, designadamente: Connectivism and Connective Knowledge course (CCK08); Digital Storytelling (DS106) e o Learning Analytics and Knowledge (LAK12) (Rodriguez, 2012). Os xMOOC, provenientes do MIT/Stanford, são centrados nos conteúdos e com uma 
organização mais rígida, limitando a criatividade. São o modelo mais comum de MOOC e seguem um projeto de curso instrutivista no qual os objetivos de aprendizagem são predefinidos pelo instrutor (Littlejohn, 2013) e os materiais são planeados e preparados com antecedência. Os alunos assistem, essencialmente, a séries de vídeo (palestras), leem artigos recomendados e resolvem questionários (Bali, 2014). Udemy, EdX, Coursera, Udacity e FutureLearn são alguns dos exemplos das plataformas mais conhecidas (Auyeung, 2015; Downes, 2013). A Udemy foi fundada por Eren Bali e Oktay Caglar e conta, atualmente, com mais de 11 milhões de estudantes e 40.000 cursos (alguns gratuitos e outros com custos associados, dependendo do tipo de curso que se pretende frequentar). É um exemplo de uma plataforma que permite a criação de cursos sem necessidade de qualquer ligação institucional (útil para qualquer promotor a título individual que deseje desenvolver e disseminar os seus próprios MOOC). No capítulo da apresentação e discussão dos resultados caracteriza-se, de forma mais aprofundada, esta plataforma.

\section{Abordagem metodológica}

O estudo de caso é uma abordagem que se adequa a este estudo uma vez que tem como intuito explicar uma situação e descrever um objeto ou fenómeno, neste caso, as etapas que constituem o processo de desenvolvimento de um MOOC, nomeadamente no âmbito da oficina de formação e da plataforma Udemy. Para além do diário do investigador resultante da observação participante, quer através da plataforma de videoconferência Colibri Zoom, quer através da plataforma de aprendizagem INTACT e do grupo Facebook criado para o efeito, foi também construído um questionário para recolha da informação sobre o processo de desenvolvimento e avaliação do MOOC.

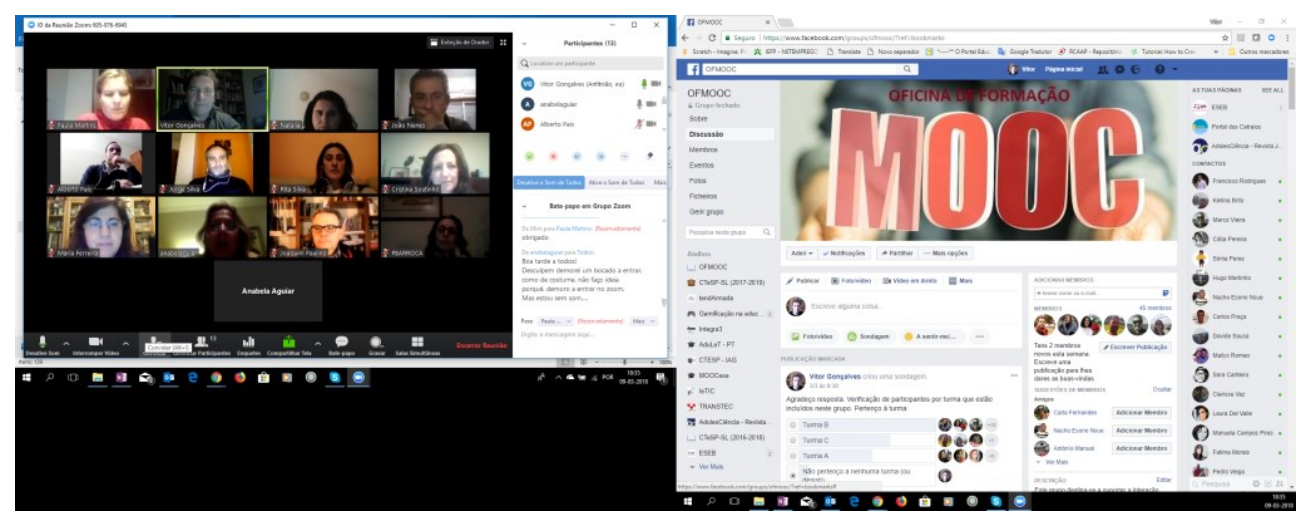

Fig. 1 MOOC sobre MOOCs e Fig. 2 MOOCs em construção 
Como o curso "MOOC sobre MOOCs e outras tecnologias educativas" (2 $2^{\mathrm{a}}$ Edição) é realizado no âmbito da formação contínua de professores, consideraram-se os educadores de infância e os professores dos ensinos básico e secundário como destinatários desta pesquisa.

\section{Desenvolvimento e resultados da oficina de formação}

A formação "MOOC: tecnologia educativa de futuro" foi realizada em modalidade de oficina de formação (25 horas síncronas e 25 horas assíncronas) verificando-se a concretização dos seguintes objetivos principais: (i) Compreender o conhecimento teórico e prático sobre os MOOC e o seu papel na sociedade digital atual; (ii) Contribuir para o crescimento do professor, tanto como profissional quanto como indivíduo; (iii) Promover o enriquecimento dos conhecimentos e competências no domínio da conceção, desenvolvimento e utilização dos MOOC pelos professores.

As sessões síncronas inerentes ao conhecimento, compreensão e discussão dos tipos, variantes e modelos dos MOOC e respetiva planificação do MOOC de cada docente decorreram nos meses de março e abril de 2018 e as sessões assíncronas de aplicação e apoio ao desenvolvimento do MOOC de cada docente decorreram nos meses de maio e junho de 2018 .

Dos 69 candidatos inscritos, registaram-se 60 formandos em três turmas de acordo com os seus interesses individuais de horário. A sessão de apresentação da oficina de formação teve a duração de 1 hora para cada uma das turmas. As sessões síncronas que se seguiram decorreram em 8 sessões de 3 horas cada, perfazendo as 25 horas síncronas online através de quatro plataformas principais: 1) Plataforma Colibri ZOOM: https://videoconfcolibri.zoom.us/j/6059766945 para a realização através de videoconferência das sessões de formação e outras reuniões em pequeno à distância; 2) Plataforma INTACT, através da disponibilização online da unidade de formação "Oficina de Formação ABC MOOC": http://www.intactschools.eu/pt-pt/course/1533/abc-mooc-uma-tecnologia-educativa-do-

futuro/; 3) MOOC sobre MOOCs e outras tecnologias educativas: curso disponibilizado através da plataforma Udemy: https://www.udemy.com/mooc-sobre-moocs-e-outrastecnologias-educativas/ que funcionava simultaneamente como ambiente de estudo da oficina de formação e exemplo de curso MOOC. 4) Grupo no Facebook: https://www.facebook.com/groups/ofmooc/ para facilitar a comunicação entre os formandos e fomentar a partilha de ideias, ferramentas associadas e esclarecimento de dúvidas.

O processo de desenvolvimento da $2^{\mathrm{a}}$ edição do MOOC caracterizou-se por um conjunto de atividades de índole tecnológica, pedagógica e didática. 
1) Registo e autenticação: o instrutor pode autenticar-se na plataforma Udemy através das redes sociais Facebook ou Google ou realizando previamente o registo (nome do instrutor, o seu email e a password para posterior acesso. De seguida, receberá no seu e-mail uma mensagem para confirmação e ativação da conta na plataforma Udemy.

2) Configurações do curso: após o registo e autenticação, o instrutor está em condições de efetuar as configurações básicas do curso, quer ao nível da definição do tipo de privacidade que pretende para o MOOC, quer ao nível das permissões de acesso (normalmente estas deverão ser atribuídas apenas aos instrutores do curso). Para além disso poderá:

2.1) Aluno-alvo: espaço para adicionar a descrição sobre o tipo de alunos e respetivos pré-requisitos em termos de ferramentas e conhecimentos que os interessados necessitam para alcançar com sucesso os objetivos e competências apresentados também neste item.

2.2) Grade curricular: espaço onde o instrutor constrói o seu curso. Podem ser utilizadas secções que permitem dividir os conteúdos e os objetos de aprendizagem a serem disponibilizados. No interior de cada secção pode ser adicionado um conjunto de itens, tais como: aulas, que podem conter vídeos, mashups e artigos; questionários de escolha múltipla; exercícios de programação (C++, C\#, HTML, JavaScript, ES6, Java, PHP5, PHP7, Python 3, Ruby e Swift 3); simulações; e tarefas. Comparativamente com a $1^{\text {a }}$ edição do curso "MOOC sobre MOOCs e outras tecnologias educativas" realizada em 2015, verificou-se uma evolução satisfatória da plataforma Udemy e, consequentemente, do próprio curso.

3) Mensagens automáticas: área para automatizar respostas e incentivar os alunos a interagir com o conteúdo, o instrutor pode escrever mensagens que, posteriormente, serão enviadas automaticamente para os alunos quando eles acederem ou concluírem o MOOC.

4) Avaliação (Análise): etapa para que o instrutor possa submeter o seu MOOC para análise por parte da Udemy com vista a averiguar se o curso se encontra dentro dos parâmetros definidos pela empresa, quer ao nível da adequação do tipo de conteúdos, quer ao nível da sua qualidade. De referir que este passo só ocorre depois de terem sido preenchidos todos os requisitos através da validação pelo algoritmo de software da Udemy.

5) Página inicial do curso: etapa que permite definir o nível de aprendizagem ou nível de dificuldade do curso (iniciante, intermediário ou especialista); o título, o subtítulo e a respetiva descrição do curso, bem como a imagem do curso (750 x 422 pixels; extensão do formato: .jpg, .jpeg, .gif ou .png) e o vídeo promocional que contribua para captar formandos para o MOOC. De referir que nesta etapa a plataforma disponibiliza um guia para criar um vídeo promocional. 
6) Legendas: etapa facultativa que facilita a compreensão dos conteúdos disponibilizados no MOOC, nomeadamente os vídeos que o instrutor inseriu na grade curricular.

7) Perfis de instrutores: o instrutor é convidado a preencher o seu perfil, incidindo, particularmente, na sua profissão, biografia, idioma nativo e links externos para redes sociais ou páginas Web. Note que o perfil do instrutor pode ser consultado por qualquer formando que participe no MOOC.

Avaliada a sua participação, podemos concluir dois aspetos principais: a participação numa oficina de formação deste tipo exige (auto)motivação e imbricação por parte dos formandos, o que explica a desistência de 26 formandos ao longo da oficina de formação.

Dos 34 formandos que concluíram esta oficina, apesar das dificuldades em conciliar a formação com o trabalho laboral, podemos resumir a sua avaliação: i) demonstraram ser pontuais e assíduos, cumprindo com empenho as tarefas propostas; ii) demonstraram predisposição ou mesmo prática no desenvolvimento das literacias emergentes e integração destas tecnologias na sua prática letiva; iii) salientaram a adequada dinâmica pedagógica, por promover o debate e a troca de experiências e saberes, bem como um excelente ambiente virtual de partilha e entreajuda que existiu entre os formandos; iv) adquiriram competências e capacidades essenciais e demonstraram sentido crítico e empreendedor na tarefa de trabalhar os próprios MOOC para formar públicos na comunidade escolar e fora dela; v) apesar de todos, sem exceção, enfatizarem as potencialidades desta modalidade de formação e dos resultados que a mesma lhes poderá vir a proporcionar num futuro próximo, salientaram que o ambiente descontraído da oficina de formação contibuiu para a compreensão de conteúdos e a aprendizagem e que a ajuda do formador foi imprescindível na sua rapidez e objetividade, o que permitiu ultrapassar algumas dificuldades; vi) destacaram ainda o espírito colaborativo que se instalou entre os formandos, quer nas sessões online síncronas, quer durante a partilha dos cursos foi uma das partes do trabalho mais enriquecedora.

Em suma, parece ser que a formação à distãncia constiui uma mais-valia na disseminação da formação de professores que permite, independentemente da sua localização geográfica, frequentar um maior leque de oferta formativa que não estaria disponível localmente.

\section{Considerações Finais}

Educar é a construção de um percurso para o amanhã, quer a nível individual, quer a nível coletivo. Os MOOC podem contribuir para o desenvolvimento das sociedades e das comunidades educativas, em geral, e para a formação contínua dos professores, em particular. 
O desenvolvimento e avaliação da oficina de formação "MOOC: uma tecnologia educativa do futuro" e do respetivo MOOC e demais plataformas que a suportaram, permitiu responder a isso mesmo através da caracterização do processo de desenvolvimento do "MOOC sobre MOOCs e outras tecnologias educativas" na plataforma Udemy e, principalmente, através da avaliação da 2. ${ }^{a}$ edição deste tipo de formação. Neste sentido, através da análise dos resultados, foi possível determinar que o processo de desenvolvimento de um MOOC pode ser caracterizado em três dimensões - tecnologia, pedagogia e conteúdo, que contribuirão decisivamente para um processo de ensino e de aprendizagem de qualidade.

O facto de ser totalmente online impôs, necessariamente, uma estrutura e uma organização muito adequada, para que o acompanhamento e participação pudesse ocorrer com normalidade através das plataformas referidas. Todas as sessões síncronas tiveram um momento final de conclusões, favorecendo uma visão interdisciplinar e holística, sem descurar a aplicabilidade destas ferramentas digitais às áreas de interesse de cada participante.

Destaca-se que, para além da utilização da plataforma de videoconferência Colibri Zoom se ter mostrado muito adequada, as sessões síncronas beneficiaram muito com a participação de colegas da edição anterior e investigadores da área dos MOOC.

Não podemos também deixar de destacar que, apesar de termos consciência de que os MOOC da Udemy possuem uma organização mais rígida com objetivos de aprendizagem bem definidos, estamos convictos que podem dar um contributo relevante para a formação contínua de professores, tal como enfatizam os resultados apresentados. Ou seja, os aspetos positivos desta tipologia de cursos é um excelente complemento à formação contínua de professores em modalidade e-learning/b-learning e não apenas porque o acesso à plataforma Udemy, e a este curso em particular, é gratuito para formadores ou professores.

\section{Referências}

Auyeung, V. (2015). Review: To MOOC or not to MOOC: Issues to consider for would-be MOOC academic leads. Higher Education Research Network Journal, 9, 64-71.

Bali, M. (2014). MOOC pedagogy: gleaning good practice from existing MOOCs. Journal of Online Learning and Teaching, 10(1), 44.

Baylor, A. L. \& Ritchie, D. (2002). What factors facilitate teacher skill, teacher morale, and perceived student learning in technology-using classrooms? C\&E, 39(4), 395-414.

Codarin, L. (2012). Impacto de los MOOC en la formación corporativa. Retrieved from http://www.slideshare.net/lcodarin/mooc-leandro-2012 
Daradoumis, T., Bassi, R., Xhafa, F., \& Caballé, S. (2013). A review on massive e-learning (MOOC) design, delivery and assessment. In P2P, Parallel, Grid, Cloud and Internet Computing (3PGCIC), 2013 Eighth International Conference (pp. 208-213). IEEE.

Diário da República. (2009). Decreto-Lei n.o 731/2009 de 7 de julho (Vol. Diário da República I Série-A). Lisboa: Ministério da Educação. Lisboa.

Downes, S. (2012). Massively Open Online Courses Are "Here to Stay.”.

Downes, S. (2013). What the "x" in “xMOOC"stands for. Retrieved October, 27, 2017.

Littlejohn, A. (2013). Understanding massive open online courses. CEMCA EdTech Notes.

Ma J.D., Lee, K.C. \& Kuo, J.M. (2013). A massive open online course on pharmacogenomics: not just disruptive innovation but a possible solution. Pharmacogenomics, 14(10), 1125-1127.

Mattar, J. (2013). Aprendizagem em ambientes virtuais: teorias, conectivismo e MOOCS. São Paulo: TECCOGS-PUC/SP, (7), 21-40.

Monterrey, T. de. (2014). Edu Trends Report. Observatorio de Innovación Tecnológica del Tecnológico de Monterrey. Retrieved from http://observatory.itesm.mx/edutrendsmooc/

Monterrey, T. de. (2017). Radar de Innovación Educativa 2017. Observatorio de Innovación Tecnológica del Tecnológico de Monterrey. Retrieved from Monterrey, México: https://observatorio.itesm.mx/radar-de-innovacin-educativa-2017

Penteado, M., \& Borba, M. C. (2000). A informática em ação: formação de professores, pesquisa e extensão. São Paulo, Olho D’Àgua.

Rodriguez, C. O. (2012). MOOCs and the AI-Stanford like courses: Two successful and distinct course formats for massive open online courses. European Journal of Open, Distance and ELearning, 15(2).

Shah, D. (2018). 6 Biggest MOOC Trends of 2017. 2017 MOOC Roundup Series. Class Central. Retrieved from https://www.class-central.com/report/6-biggest-mooc-trends-2017/

Siemens, G. (2013). Massive open online courses: Innovation in education. Open Educational Resources: Innovation, Research and Practice, 5.

Torres, D. (2013). Reflexiones y primeiros resultados de MOOCs em Iberoamerica: UNEDCOMA y UNX. In: Revista Iberoamericana Tecnologia Educativa, v.2, n. 1.

Watters, A. (2012). Top Ed-Tech Trends of 2012: MOOCs. Hack Education. 